\title{
Síndrome de Crigler-Najjar tipo 2 - um caso atípico
}

\author{
Crigler-Najjar syndrome type 2 - an atypical case
}

\author{
Filipa Raposo ${ }^{1}$, Margarida Reis Morais ${ }^{1}$, Marina Pinheiro' ${ }^{1}$, Mariana Costa ${ }^{1}$, Isabel Martinho ${ }^{2}$, \\ Sérgio Mendanha², Miguel Salgado ${ }^{3}$
}

${ }^{1}$ Interna Complementar de Pediatria, Serviço de Pediatria da Unidade Local de Saúde do Alto Minho, Viana do Castelo, Portugal; ${ }^{2}$ Assistente Hospitalar Graduado de Pediatria, Serviço de Pediatria da Unidade Local de Saúde do Alto Minho, Viana do Castelo, Portugal; ${ }^{3}$ Assistente Hospitalar de Pediatria, Serviço de Pediatria do Centro Hospitalar do Médio Tejo, Torres Novas, Portugal.

\section{RESUMO}

Objetivos: Os autores descrevem um caso de síndrome de Crigler-Najjar tipo 2, um distúrbio hereditário do metabolismo da bilirrubina, resultante de um déficit parcial da enzima uridino-difosfo-glicuronil-transferase (UDPG-T).

Descrição do caso: Uma lactente de etnia asiática foi internada com cinco semanas de vida por icterícia persistente desde o nascimento, com relato materno de agravamento progressivo. Ao exame objetivo apresentava-se ativa, reativa, ictérica e com ligeira hipotonia axial. A investigação complementar mostrou um aumento da bilirrubina total $(32,94 \mathrm{mg} / \mathrm{dL})$, com bilirrubina direta de $0,94 \mathrm{mg} / \mathrm{dL}$, e o estudo molecular revelou duas mutações em heterozigotia no gene UGT1A1 (c.211G>A e c.1456T>G), resultado compatível com síndrome de Crigler-Najjar tipo 2. Foi submetida a fototerapia intensiva em associação com quelante dos ácidos biliares, com resposta parcial. Após conhecimento do resultado do estudo molecular iniciou fenobarbital, ocorrendo normalização dos valores de bilirrubina após duas semanas.

Conclusões: A síndrome de Crigler-Najjar tipo 2, embora fenotipicamente semelhante ao tipo 1, tem tratamento e prognóstico diferentes. Neste caso, a apresentação neonatal precoce e os valores de bilirrubina muito elevados, que não cediam totalmente à fototerapia intensiva, levaram inicialmente à suspeita de síndrome de Crigler-Najjar tipo 1, que é a forma mais grave. Os autores pretendem com este caso alertar para uma causa rara de icterícia, que não teve a apresentação típica.

DESCRITORES: ICTERÍCIA NEONATAL; BILIRRUBINA; SÍNDROME DE CRIGLER-NAJJAR; FOTOTERAPIA.

\section{ABSTRACT}

Aims: The authors describe a case of Crigler-Najjar syndrome type 2, an inherited disorder of bilirubin metabolism resulting from a partial deficit of the enzyme uridine- diphospho-glucuronyl transferase (UDPG-T).

Case description: A female infant of Asian ethnicity was admitted with five weeks of age by persistent jaundice since birth, with maternal report of progressive worsening. Upon physical examination the patient was active, reactive, and jaundiced, with mild axial hypotonia. Complementary examination showed increase in total bilirubin $(32.94 \mathrm{mg} / \mathrm{dL})$, with direct bilirubin of $0.94 \mathrm{mg} / \mathrm{dL}$, and molecular study revealed two heterozygous mutations in the UGT1A1 gene (c.211G $>$ A and c. $1456 \mathrm{~T}>\mathrm{G}$ ), consistent with Crigler-Najjar syndrome type 2. She was submitted to intensive phototherapy in combination with bile acid chelator, with a partial response. After reading the results of molecular studies, phenobarbital was started, leading to normal levels of bilirubin in two weeks.

Conclusions: Crigler-Najjar syndrome type 2, although phenotypically similar to type 1, has different prognosis and treatment. In this case, early neonatal presentation and very high bilirubin values not fully yielded to intensive phototherapy, initially raised the suspicion of CriglerNajjar syndrome type 1, which is the most severe form of the syndrome. With this report, the authors wish to draw attention to a rare cause of jaundice, which did not have its typical course.

KEY WORDS: JAUNDICE, NEONATAL; BILIRUBIN; CRIGLER-NAJJAR SYNDROME; PHOTOTHERAPY.

Recebido em fevereiro de 2014; aceito em junho de 2014. 


\section{INTRODUÇÃO}

A icterícia resultante do aumento da bilirrubina indireta pode ser secundária a várias etiologias: multifactorial (icterícia fisiológica ou do desenvolvimento), hemolítica (congênita ou adquirida) e não hemolítica. Formas de etiologia não hemolítica incluem a icterícia do leite materno, a icterícia associada à amamentação, condições ligadas ao aumento da circulação enterohepática, síntese aumentada de bilirrubina e deficiência na captação/conjugação da bilirrubina por defeitos enzimáticos, como síndrome de Crigler-Najjar (SCN) tipo 1 e 2 e Síndrome de Gilbert. ${ }^{1-4}$

A SCN e a Síndrome de Gilbert são alterações hereditárias do metabolismo da bilirrubina, sendo a última muito mais frequente, benigna, e de apresentação mais tardia, geralmente durante a adolescência. ${ }^{5} \mathrm{~A}$ SCN é mais rara, grave e leva a valores de bilirrubina total mais elevados. O tipo 2 é fenotipicamente semelhante ao tipo 1 , mas com tratamento e prognóstico marcadamente diferentes. A confirmação diagnóstica é feita pelo estudo genético. ${ }^{4}$

Relata-se um caso de SCN tipo 2, que não teve a apresentação típica, com o objetivo de alertar para o reconhecimento precoce dessa causa rara de hiperbilirrubinemia indireta. Foi deferido o parecer da Comissão de Ética para a Saúde da Unidade Local de Saúde do Alto Minho para a publicação do caso clínico, no dia 26 de fevereiro de 2014.

\section{DESCRIÇÃO DO CASO}

Lactente do sexo feminino, de cinco semanas de vida, etnia asiática, foi referenciada ao serviço de urgência de Pediatria por icterícia persistente desde o nascimento, com relato materno de agravamento na última semana. A mãe negava recusa alimentar, acolia, sonolência excessiva ou outro tipo de alteração do comportamento.

A paciente foi resultante de uma gestação vigiada sem intercorrências, sorologias maternas sem anormalidades, mãe com grupo sanguíneo $A B, R h$ positivo, parto eutócico às 39 semanas e com somatometria ao nascimento adequada à idade gestacional. Alimentada com leite materno exclusivo com intervalos de duas horas, com boa evolução ponderal. Os antecedentes familiares eram irrelevantes, sendo negada consanguinidade e história familiar de icterícia.

Ao exame objetivo apresentava-se ativa, reativa, ictérica e com ligeira hipotonia axial. A restante avaliação clínica não demonstrou outras alterações. Os exames de laboratório demonstraram aumento da bilirrubina total $(32,94 \mathrm{mg} / \mathrm{dL})$, com bilirrubina direta de $0,94 \mathrm{mg} / \mathrm{dL}$. Outros exames complementares efetuados incluíram o grupo sanguíneo $(\mathrm{A}, \mathrm{Rh}$ positivo), hemograma com reticulócitos, reação de Coombs direta, proteína $\mathrm{C}$ reativa, aspartato aminotransferase, alanina aminotransferase, gama glutamil transpeptidase, fosfatase alcalina, albumina sérica, proteínas séricas totais, lactato desidrogenase (LDH), estudo da coagulação, gasometria arterial, cultura de urina e ecografia abdominal, todos sem alterações.

Devido ao quadro de icterícia não conjugada severa de apresentação neonatal precoce, foi internada no serviço de Pediatria por suspeita de SCN tipo 1, tendo sido pedido estudo molecular para o gene UGT1A1, para confirmação diagnóstica. Iniciou fototerapia intensiva (12-16 horas/dia), reforço da hidratação e carbonato de cálcio por via oral $250 \mathrm{mg} / \mathrm{dia}$. Com a terapêutica instituída os valores de bilirrubina variaram entre $18,1 \mathrm{mg} / \mathrm{dL}$ no segundo dia de tratamento e $12,5 \mathrm{mg} / \mathrm{dL}$ no $15^{\circ}$ dia de tratamento.

No $21^{\circ}$ dia de hospitalização foi obtido o resultado do estudo molecular, que revelou duas mutações em heterozigotia no gene UGT1A1: c.211G >A (p.Gly71Arg) e c.1456T $>$ G (p.Tyr486Asp), resultado compatível com SCN tipo 2. Nessa data suspendeu-se a fototerapia e foi iniciado o fenobarbital $2 \mathrm{mg} / \mathrm{kg} /$ dose em duas doses diárias, com diminuição progressiva dos valores de bilirrubina.Teve alta ao $30^{\circ}$ dia de hospitalização, assintomática, apresentando bilirrubina total de $5,1 \mathrm{mg} / \mathrm{dL}$, e foi encaminhada para consulta de Gastroenterologia Pediátrica e Genética Médica. Atualmente está sem medicação, assintomática, com bom desenvolvimento psicomotor e a última dosagem de bilirrubina total foi de $1,02 \mathrm{mg} / \mathrm{dL}$.

\section{DISCUSSÃO}

A SCN é uma patologia hereditária do metabolismo da bilirrubina, de transmissão autossômica recessiva, resultante de um déficit da enzima uridino-difosfoglucuronil-transferase (UDPG-T), que pode ser total (SCN tipo 1) ou parcial (SCN tipo 2). É uma doença rara, com uma incidência anual de um caso por milhão de nascimentos e mais frequente em populações com altas taxas de consanguinidade. A SCN tipo 1 foi descrita pela primeira vez por Crigler e Najjar em 1952, com o relato de seis casos, em três famílias, de icterícia grave nos primeiros dias de vida, em que se verificava um predomínio de bilirrubina indireta, vindo as crianças a falecer por kernicterus antes dos dois anos de idade. O tipo 2 foi descrito em 1962 por Arias. ${ }^{1-4,6}$ 
O tipo 1 é a forma mais severa da síndrome e é consequência da deleção, mutação ou inserção em qualquer um dos cinco éxons que constituem o gene UGT1A1 (UDP glucuronosyltransferase 1 family, polypeptide A1), do que resulta ausência da enzima UDPG-T. ${ }^{7} \mathrm{Na}$ maioria dos casos apresenta-se nos primeiros dias de vida com hiperbilirrubinemia indireta, que geralmente ultrapassa os $20-25 \mathrm{mg} / \mathrm{dL}$ e pode atingir os $50 \mathrm{mg} / \mathrm{dL}$, levando a um risco elevado de kernicterus. ${ }^{4}$

O tratamento do tipo 1 consiste em fototerapia oito a dezesseis horas por dia, que converte a fração IXalfa-ZZ da bilirrubina indireta em isômeros que podem ser excretados na bílis. A fototerapia pode ser associada com quelantes biliares (carbonato de cálcio e colestiramina) e plasmaferese ${ }^{4,8,9} \mathrm{O}$ objetivo é manter os níveis de bilirrubina indireta abaixo de $20 \mathrm{mg} / \mathrm{dL}$. O único tratamento curativo é o transplante hepático. ${ }^{10}$ Têm sido desenvolvidos estudos com terapia genética em ratos, com resultados animadores. ${ }^{6,4}$ Apesar de todos os esforços, o prognóstico da SCN tipo 1 é sombrio, sendo o kernicterus a principal causa de morte. ${ }^{11-13}$ Mesmo com o tratamento adequado, quase todos os doentes com SCN tipo 1 desenvolvem sequelas neurológicas permanentes. ${ }^{2,4}$ Sem tratamento adequado, a maioria dos doentes morre pelos 15 meses de vida. ${ }^{2}$

A SCN tipo 2 resulta da substituição de um aminoácido, o que diminui a atividade catalítica da enzima UDPG-T, levando a uma deficiência parcial desta. ${ }^{7}$ A apresentação é em regra mais tardia, estando descrito que apenas metade dos pacientes ficam ictéricos no primeiro ano de vida, e o aparecimento de encefalopatia é raro. ${ }^{1,4}$ Quando se manifesta no período neonatal, a hiperbilirrubinemia indireta raramente ultrapassa os $20-25 \mathrm{mg} / \mathrm{dL}^{4}$

O tratamento do tipo 2 inclui fenobarbital, $2 \mathrm{mg} /$ $\mathrm{kg} /$ dose (2 a 3 doses/dia), que atua como indutor enzimático, fototerapia e plasmaferese (quando há uma elevação abrupta da bilirrubina). ${ }^{4}$ A terapia genética e o transplante hepático ou de hepatócitos estão reservados para os casos refratários ao tratamento. $\mathrm{O}$ uso do fenobarbital reduz os níveis de bilirrubina indireta em $25 \%$ e também permite a distinção entre o tipo 1 e o tipo 2, uma vez que no primeiro não há qualquer resposta à sua administração. ${ }^{4,8} \mathrm{~A} \mathrm{SCN}$ tipo 2 tem uma evolução clínica mais benigna e é raro o desenvolvimento de kernicterus. Quando este ocorre, geralmente os doentes sofreram algum tipo de intercorrência, como infeção, ou outro tipo de stress. ${ }^{2,4}$

O diagnóstico de $\mathrm{SCN}$ é confirmado pelo estudo molecular do gene UGT1A1.,2,4,6, São descritas mais de 50 mutações, principalmente nutações, inserções e deleções da região 2 q37 do cromossoma 2 . $^{14-16}$ Caso se confirme o diagnóstico, é importante fazer aconselhamento genético aos pais. ${ }^{1}$ A bilirrubina resultante da decomposição do grupo heme é um composto tóxico insolúvel em água. A glucorinização hepática da bilirrubina insolúvel é catalizada pela enzima UDPG-T, o que é essencial para a sua excreção na bilis. ${ }^{14}$ As mutações no gene UGTA1A1 levam à ausência total ou parcial da atividade desta enzima, resultando em aumento na concentração de bilirrubina indireta.

No caso relatado, a clínica, os valores de bilirrubina, hematócrito e reticulócitos, o esfregaço de sangue periférico e a LDH normais, bem como a reação de Coombs direta negativa, permitiram excluir a icterícia fisiológica, a associação ao leite materno e à amamentação, e a icterícia de causa hemolítica. Considerou-se como provável causa para este quadro clínico a icterícia secundária a um defeito da captação e/ou conjugação da bilirrubina (SCN ou Síndrome de Gilbert). Na Síndrome de Gilbert, os valores de bilirrubina são bastante inferiores, pelo que se excluiu esta hipótese diagnóstica. A apresentação precoce e o valor muito alto de bilirrubina indireta eram mais sugestivos da $\mathrm{SCN}$ tipo 1, pois está descrito que na SCN tipo 2 a apresentação não é tão precoce e os valores de bilirrubina raramente ultrapassam os 20-25 mg/dl. ${ }^{2,4}$ Sendo a SCN tipo 1 a hipótese mais provável, decidiu-se iniciar de imediato fototerapia intensiva 12 a 16 horas por dia, associada ao carbonato de cálcio. Com a terapêutica inicial, foi verificada uma redução dos valores de bilirrubina total em cerca de $60 \%$. Esta resposta positiva ao tratamento instituído fez com que não se optasse por tratamentos mais agressivos, como a plasmaferese.

$\mathrm{O}$ estudo molecular do gene UGT1A1, efetuado por amplificação por PCR e sequenciação direta de toda a região codificante, incluindo regiões intrônicas adjacentes do gene, foi disponibilizado no $21^{\mathrm{o}}$ dia de hospitalização. O estudo demonstrou duas mutações em heterozigotia: c.211G $>$ A (p.Gly71Arg) e c.1456T $>$ G (p.Tyr486Asp), o que confirmou o diagnóstico de SCN, entretanto do tipo 2, e estabeleceu a sua etiologia molecular. Ambas as mutações são conhecidas, tendo já sido descritas em outros doentes. A mutação c.211G $>$ A (p.Gly71Arg) está associada a uma redução da atividade enzimática de cerca de $47 \%$, sendo este alelo conhecido por UGT1A1*6. ${ }^{17,18}$ A mutação c. 1456T $>\mathrm{G}$ (p.Tyr486Asp), correspondente ao alelo UGT1A1*7, foi descrita como causadora da doença. ${ }^{19}$ Tem sido sugerido por diversos estudos que 
a associação da mutação homozigotica (p.Tyr486As), com a mutação em homozigótica/heterozigotica do p.Gly71 Arg, é sempre expressa nos casos de SCN tipo 2 na população asiática. ${ }^{20} \mathrm{Na}$ data em que se obteve o resultado do estudo molecular foi iniciado fenobarbital, com normalização dos valores de bilirrubina em poucas semanas.

O não uso inicial de fenobarbital será talvez a decisão mais controversa e passível de discussão. Embora esteja descrito que este fármaco pode ajudar na diferenciação entre os dois tipos de $\mathrm{SCN}$, não é menos verdade que o uso de fenobarbital não é isento de riscos e, segundo a literatura, associa-se a atraso cognitivo, ${ }^{21} \mathrm{o}$ que poderia comprometer ainda mais o neurodesenvolvimento de uma criança que pela doença de base se encontrava já em risco neurológico. Por outro lado, uma hiperbilirrubinemia tão marcada teria de ser sempre tratada com outros métodos, tais como a fototerapia intensiva e os quelantes; assim, mesmo que o fenobarbital fosse também adicionado, não seria possível medir qual a contribuição individual de cada tratamento na diminuição da bilirrubina e inferir da eficácia ou não do fármaco. Estes dados, aliados à grande probabilidade inicial de se tratar de uma SCN tipo 1 , levaram à decisão médica de não administrar o fenobarbital inicialmente, até ser possível avaliar a resposta às terapêuticas instituídas e concluir o estudo genético.

No caso apresentado, embora os valores de bilirrubina total tenham atingido valores muito elevados $(32,94 \mathrm{mg} / \mathrm{dL})$, a resposta à terapêutica instituída foi boa, pelo que não se verificaram até à data indícios de sequelas neurológicas. Este fato está de acordo com dados publicados na literatura, que referem um curso mais benigno com menor número de sequelas neurológicas na SCN tipo 2.2,4

Para concluir, a SCN é uma patologia rara, em que o diagnóstico deve ser feito o mais precocemente possível, de forma a evitar o aparecimento de kernicterus e suas respetivas sequelas. Devemos pensar nessa doença quando estamos perante um recémnascido/lactente ictérico, com valores muito elevados de bilirrubina predominantemente indireta, com função hepática normal e sem sinais de hemólise. Este caso alerta para uma apresentação atípica da SCN do tipo 2, que inicialmente simulou a SCN tipo 1 . Salienta-se a importância do estudo molecular, que contribuiu para um diagnóstico preciso, indicando o tratamento adequado que obteve boa resposta clínica.

\section{REFERENCIAS}

1. Kliegman MR, Behrman ER, Jenson BH, Stanton FB. Nelson Tratado de Pediatria. 18 ${ }^{\underline{a}}$ ed. Rio de Janeiro: Saunders Elsevier; 2009.

2. Hisham N, Praveen KR. Unconjugated Hyperbilirrubinemia [Internet]. Medscape Website [updated 2013 Jun; cited 2013 Set]. Available from: http://emedicine.medscape.com

3. Crigler JF, Najjar VA. Congenital familial nonhemolytic jaundice with kernicterus. Pediatrics. 1952;10:169-79.

4. Chowdhury-Roy N, Chowdhury-Roy J. Crigler-Najjar Syndrome [Internet]. UpToDate Website [updated 2013 Apr; cited 2013 Set]. Available from: http://www.uptodate.com

5. Chowdhury-Roy N, Chowdhury-Roy J. Gilbert syndrome and unconjugated hyperbilirubinemia due to bilirubin overproduction. UpToDate Website [updated 2009 Fev; cited 2013 Set]. Available from: http://www.uptodate.com

6. Jansen PL. Diagnosis and management of Crigler-Najjar Syndrome. Eur J Pediatr. 1999;158 Suppl 2:S89-94.

7. Bosma PJ, Goldhoorn B, Oude-Elferink RPJ, Sinaasappel M, Oostra BA, Jansen PLM. A mutation in bilirubin uridine 5'-diphosphateglucuronosyltransferase isoform-1 causing Crigler-Najjar syndrome type-I. Gastroenterology. 1993;105:216-20.

8. Torrecilla B, Atance E, Grande C, Fernández M. Síndrome de Crigler-Najjar: diagnóstico y tratamiento. Pediatr (Barc). 2006;65(1):73-8.

9. Nydegger A, Bednarz A, Hardikar W. Use of daytime phototherapy for Crigler-Najjar disease. J Pediatric Child Health. 2005;41(7):387-9.

10. Pareja E, Ribes C, Gómez-Lechón MJ, Cortes M, Vila JJ, Dalmau J, Ibánez V, Polo V, Castell JV, Mir J. Terapia celular hepática en el tratamiento de las metabolopatías congénitas en ninos. An Pediatr (Barc). 2013;79(6):390.e1-390.e8.

11. Springer S, Annibale D. Kernicterus [Internet]. Medscape Website [updated 2012 Apr; cited 2014 Jan]. Available from: http://emedicine. medscape.com

12. Ip S, Lau J, Chung M, Kulig J, Sege R, Glicken S, O’Brien R. Hyperbilirrubinemia and kernicterus: 50 years later. Pediatrics. 2004;114: 263-4.

13. Round T. Bilirrubin brain toxicity. J Perinatology. 2001;21:S48-51.

14. Costa E. Hematologically important mutations: Bilirubin UDP-glucuronosyltransferase gene mutations in Gilbert and Crigler-Najjar syndromes. Blood Cells Mol Dis. 2006;36:77-80.

15. Iolascan A, Meloni A, Cappolla B, Rosatelli M. Crigler Najjar syndrome type II resulting from three different mutations in the bilirrubin uridine 5 -diphosphate-glucuronosyltransferase (UGT1A1) gene. J Med Genet. 2000;37:712-3. 
16. Bosma P, Chowdhury JR, Huang TJ, Lahiri P, Elferink RP, Van Es HH, Lederstein M, Whitington PF, Jansen PL, Chowdhury NR. Mechanisms of inherited deficiencies of multiple UDP-glucuronosyl transferase isoforms on two patients with Crigler Najjar syndrome type I. Faseb Journal.1992;6:2859-63.

17. Kang TW, Kim HJ, Ju H, Kim JH, Jeon YJ, Lee HC, Kim KK, Kim JW, Lee S, Kim JY, Kim SY, Kim YS. Genome-wide association of serum bilirubin levels in Korean population. Hum Mol Genet. 2010;19:3672-8.

18. Teng HC, Huang MJ, Tang KS. Combined UGT1A1 and UGT1A1 variant alleles are associated with increased risk of Gilbert's Syndrome in Taiwanese adults. Clin Genet. 2007;72:321-8.

19. Di YM, Chan E, Wei MQ, Liu JP, Zhou SF. Prediction of deleterious non synonymous single-nucleotide polymorphisms of human uridine diphosphate glucuronosyltransferase genes. AAPS J. 2009;11:469-80.

20. Ko JS, Chang JY, Moon JS, Yang HR, Seo JK. Molecular Analysis of the UGT1A1 Gene in Korean Patients with Crigler-Najjar Syndrome Type II. Pediatr Gastroenterol Hepatol Nutr. 2014;17(1):37-40.

21. Loring DW, Meador J K. Cognitive side effects of antiepileptic drugs in children. Neurology. 2004;62(6):872-7. 